\title{
Very compact photonic crystal resonant cavity for all optical filtering
}

\author{
Hamed Seif-Dargahi • Mahdi Zavvari • \\ Hamed Alipour-Banaei
}

Received: 21 May 2014/ Accepted: 9 August 2014/Published online: 11 September 2014

(C) The Author(s) 2014. This article is published with open access at Springerlink.com

\begin{abstract}
In this paper, an all-optical filter is proposed which employs a resonant cavity localized between input and output waveguides as wavelength selecting part of the filter. We study the impact of different parameters on the filtering of the structure using plane wave expansion and finite difference time domain methods. We also study the effect of the output waveguide position on the performance of the filter and results show that the proper position of the output waveguide is straight to the center of the resonant cavity. The initial form of filter is capable of selecting the optical waves at $\lambda=1,554.2 \mathrm{~nm}$ and the transmission efficiency of the filter is obtained about $100 \%$. The total footprint of the filter is $<76 \mu \mathrm{m}^{2}$. High transmission efficiency, simplicity of design and compact size are the most significant advantages of filter.
\end{abstract}

Keywords Photonic crystals - All optical filter . Resonant cavity $\cdot$ Kerr effect

H. Seif-Dargahi

Department of Electrical Engineering,

Ahar Branch, Islamic Azad University, Ahar, Iran

M. Zavvari $(\square)$

Department of Electrical Engineering,

Urmia Branch, Islamic Azad University, Urmia, Iran

e-mail: m.zavvari@iaurmia.ac.ir

H. Alipour-Banaei

Department of Electrical Engineering,

Tabriz Branch, Islamic Azad University, Tabriz, Iran

\section{Introduction}

Since the invention of photonic crystals $(\mathrm{PhC})$ in 1987, a new field of research has been created for optical device scientists. PhCs mainly are composed of two different dielectric materials with different refractive indices [1-4]. The arrangement of these two dielectric materials is such that the distribution of refractive index is periodic. According to the periodicity, these artificial structures can be demonstrated in three categories: 1D, 2D and 3D PhCs $[5,6]$. Similar to semiconductor devices, the periodicity in distribution of refractive index results in a wavelength band at which no photons or electromagnetic waves are allowed to propagate inside the $\mathrm{PhC}[7,8]$. This wavelength band is called photonic band gap (PBG). Due to critical importance of PBG, so many studies have been done on properties of PBG [7, 9-12]. The studies showed that PBG depends on the refractive indices of dielectric materials and the structural parameters of PhCs. The characteristics of PBG can be used for designing optical reflectors [13], optical band reject filters [14], optical filters [12], and optical demultiplexers $[15,16]$.

Optical filters play a crucial role in all optical communication networks. Removing noise and unwanted waves from the channel and separating closely spaced optical channels in wavelength division multiplexing (WDM) systems are the main applications of optical filters.

Resonant cavities [17] and ring resonators [18, 19] are the most popular structures used for designing all optical photonic crystal based filters. Alipour et al. [17] studied the filtering properties of an L4 resonant cavity. They proposed an optical filter using an L4 resonant cavity inside a 2D hexagonal photonic crystal structure composed of air holes in a dielectric substrate. The transmission efficiency and quality factor of their filter was about $75 \%$ and 2,500, 
respectively. They showed that by changing the effective length of the resonant cavity, the output wavelength of the filter can be tuned. Another optical filter using resonant cavity based on photonic crystal structures has been proposed using a periodic structure, in which a $2 \mathrm{D}$ ThueMorse structure is used [18]. The resonant part of the filter in this work is an L4 cavity, but the transmission efficiency was much higher than the periodic structure [19].

In this paper we design a very compact filter by resonant cavity located between input and output waveguides as wavelength selecting section. High transmission efficiency, compact dimension and simplicity of design are the most significant advantages of our filter.

\section{Filter design}

The fundamental platform used for designing the proposed filter is a square lattice of dielectric rods in air. The number of rods in $x$ and $y$ directions are 23 and 11, respectively. The effective refractive index of dielectric material is 3.46 , the radius of rods, $R$, is $102 \mathrm{~nm}$ and the lattice constant of the structure, $a$, is $550 \mathrm{~nm}$. Before designing the filter we need to calculate the band structure of the fundamental $\mathrm{PhC}$ and extract its PBG region. For this purpose we employed Bandsolve simulation tool of Rsoft photonic CAD software,

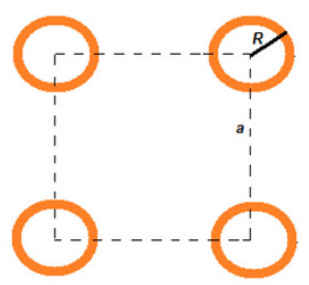

(a)

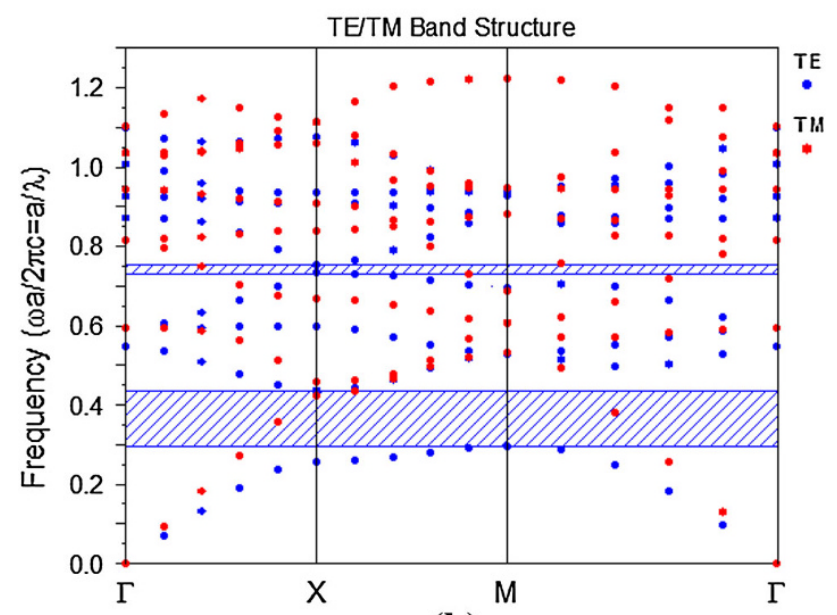

(b)

Fig. 1 a The unit cell of proposed platform, b band structure of fundamental structure

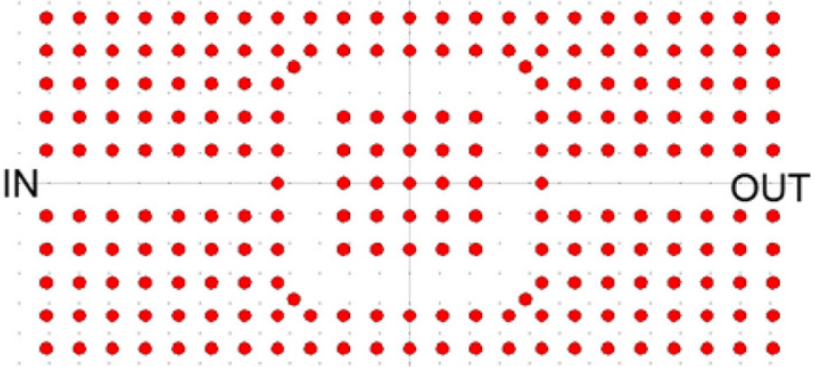

Fig. 2 The schematic of proposed filter with IN, OUT and resonator waveguides

which calculates the band structure of periodic structures based on plane wave expansion (PWE) method [20]. The band structure diagram for this $\mathrm{PhC}$ is shown in Fig. 1 with aforementioned structural parameters. According to the figure, this structure has two PBG in TM mode that is shown by dark area in the band structure of the PhC. There is no PBG for TE mode, so all the simulations will be performed in TM mode. It is evident that the PBG is in the range of $0.29<\omega a / 2 \pi c<0.43$ or in the wavelength range of $1,279 \mathrm{~nm}<\lambda<1,896 \mathrm{~nm}$.

The proposed filter is composed of one input and one output waveguide connected to each other through a resonant square cavity. This schematic of the filter was first proposed by Robinson and Nakeeran [21]. However in this paper, we study the effect of different structural parameters on the efficiency of filter to optimize its performance operation. For creating the resonant cavity we remove a $7 \times 7$ square array of rods and replace them with an $5 \times 5$ square lattice of dielectric rods. The final sketch of the filter is shown in Fig. 2. The input and output waveguides of filter are seen in figure. First, we assume that the structural parameters of the core part are the same as the fundamental structure.

\section{Simulation and results}

After finalizing the design of structure, we study its optical performance by calculation of output spectrum. For this purpose, finite difference time domain (FDTD) method is used to solve electromagnetic wave propagation equations. The output spectrum of the filter is shown in Fig. 3. In this filter the effective refractive index of dielectric material is 3.46 , the radius of rods is $102 \mathrm{~nm}$ and the lattice constant of the structure is $550 \mathrm{~nm}$. According to figure, our filter has an output mode at $1,552 \mathrm{~nm}$, near the telecom fundamental wavelength. The transmission efficiency and bandwidth of the filter are calculated as $91 \%$ and $3 \mathrm{~nm}$, respectively. Transmission efficiency is defined as the ratio of transmitted optical power to the input power. According 


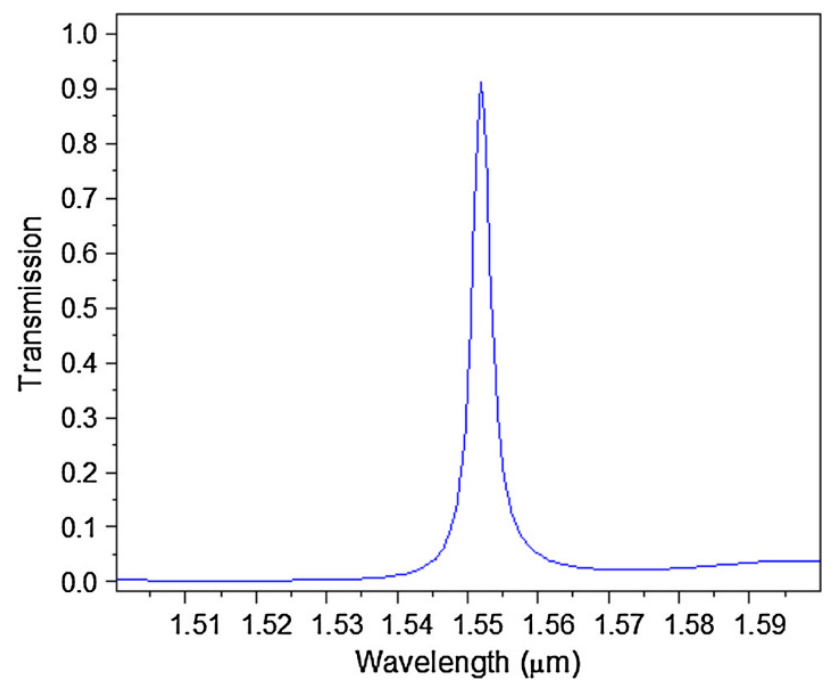

Fig. 3 The output spectrum of the proposed filter

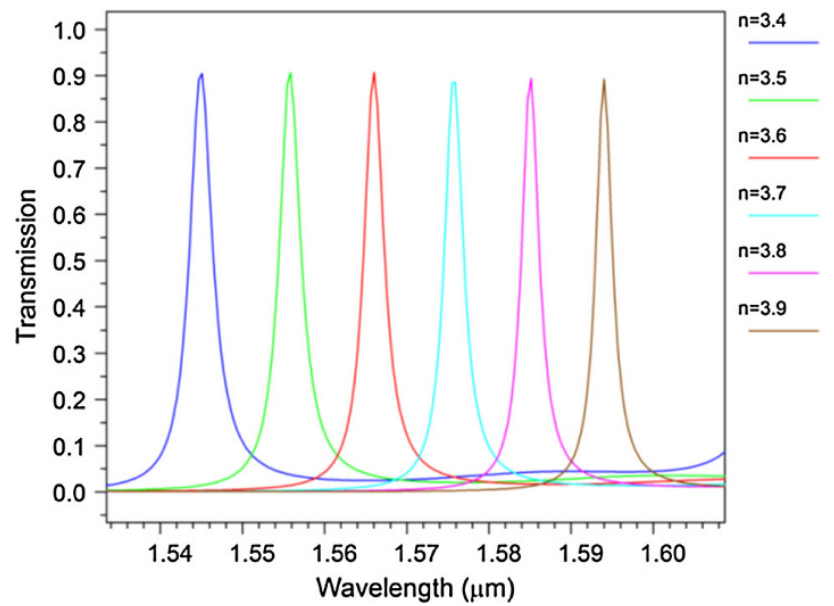

Fig. 4 The output spectra of the filter for different values of $n$ (refractive index of the rods outside the resonator). The other parameters are unchanged

to the results, the quality factor is calculated as 517 . In order to study the effect of structure parameters in the efficiency of filter, we go on by studying the effect of refractive index changes in the whole structure. The output spectra of the filter are calculated for different values of $n$ (refractive index of the rods) and are shown in Fig. 4 while the other parameters are assumed unchanged. The range of refractive index corresponds to the most diversity of dielectrics. Results show that by increasing the refractive index, the output wavelength shifts to higher values but its peak remains unchanged. The detailed specifications of the output wavelengths for different values of $n$ are listed in Table 1 . According to the table, the quality factor improves as the refractive index gets higher values.
Table 1 Significant parameters of the proposed filter for different values of $n$

\begin{tabular}{lllll}
\hline$n$ & $\lambda(\mathrm{nm})$ & $\Delta \lambda(\mathrm{nm})$ & $Q$ & TE $(\%)$ \\
\hline 3.4 & $1,545.1$ & 3.4 & 454 & 90 \\
3.5 & $1,555.8$ & 3.1 & 501 & 90 \\
3.6 & $1,566.1$ & 2.9 & 540 & 90 \\
3.7 & $1,575.9$ & 2.7 & 583 & 89 \\
3.8 & $1,585.2$ & 2.5 & 634 & 90 \\
3.9 & $1,594.1$ & 2.3 & 693 & 89 \\
\hline
\end{tabular}

$T E$ Transmission efficiency

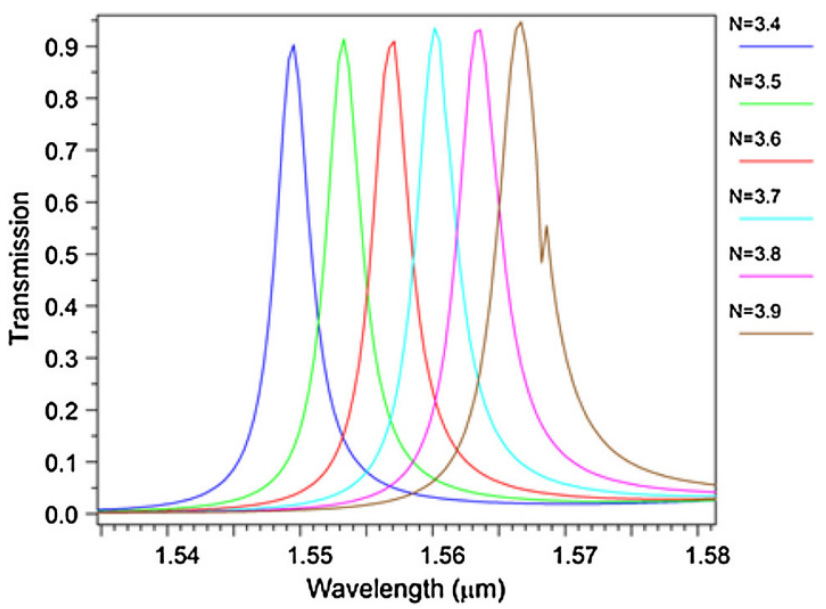

Fig. 5 The output spectra of the filter for different values of $N$ (refractive index of the rods inside core)

Table 2 Significant parameters of the proposed filter for different values of $N$

\begin{tabular}{lllll}
\hline$N$ & $\lambda(\mathrm{nm})$ & $\Delta \lambda(\mathrm{nm})$ & $Q$ & TE $(\%)$ \\
\hline 3.4 & $1,549.5$ & 3 & 516 & 91 \\
3.5 & $1,553.2$ & 3.3 & 470 & 92 \\
3.6 & 1,557 & 3.4 & 457 & 91 \\
3.7 & $1,560.4$ & 3.5 & 445 & 93 \\
3.8 & $1,563.5$ & 4 & 390 & 93 \\
3.9 & $1,566.7$ & 4 & 391 & 94 \\
\hline
\end{tabular}

Another parameter which can strongly influence the transmission characteristics of filter is the refractive index of core part of the resonant cavity $(N)$. The output of the filter is shown in Fig. 5 for different values of $N$. A similar change in the output of filter is evident where the peak wavelength shifts to longer values for increased refractive index of core. It should be noticed that the $N$ is chosen such that the output wavelength adjusted around $1,550 \mathrm{~nm}$. Table 2 shows details about the effect of $N$ on the performance of filter. From the results, despite to $n$, any increase in refractive index of core lowers the quality factor. 


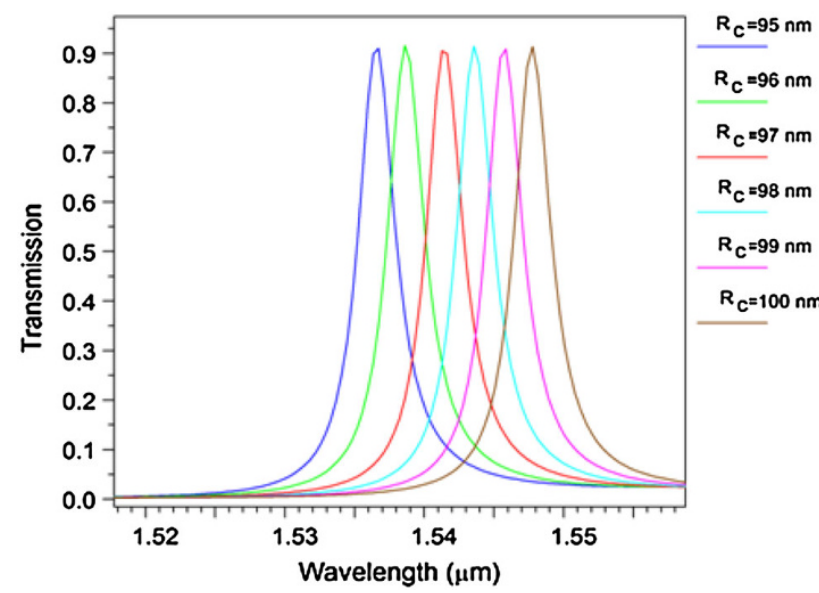

Fig. 6 The output spectra of the filter for different values of $R_{\mathrm{C}}$ (the radius of rods inside the core). Other parameters are unchanged

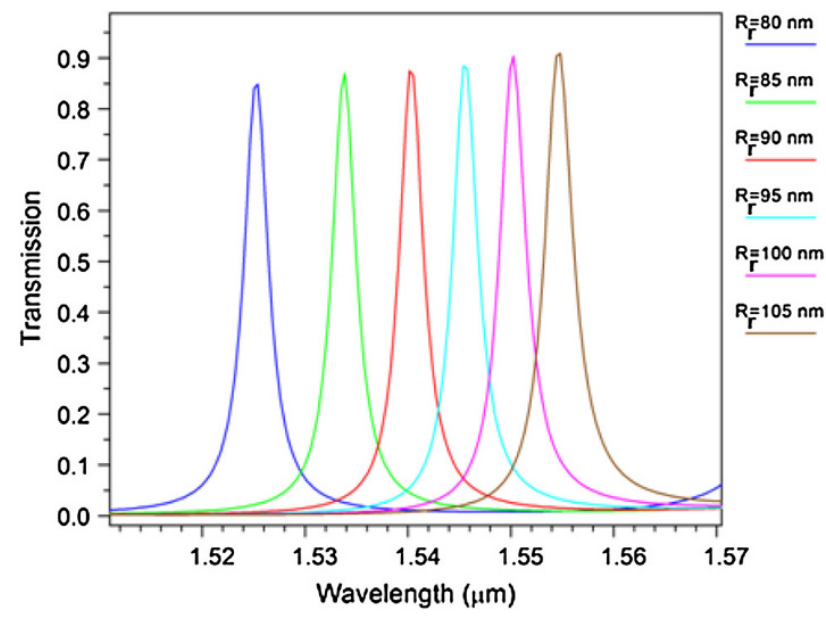

Fig. 7 The Output spectra of the filter for different values of $R_{r}$ (the radius of rods outside the core). Other parameters are unchanged

Table 3 Significant parameters of the proposed filter for different values of $R_{\mathrm{C}}$

\begin{tabular}{lllll}
\hline Radius & $\lambda(\mathrm{nm})$ & $\Delta \lambda(\mathrm{nm})$ & $Q$ & $\mathrm{TE}(\%)$ \\
\hline 95 & $1,536.5$ & 3.2 & 480 & 91 \\
96 & $1,538.7$ & 3.2 & 480 & 92 \\
97 & $1,541.5$ & 3.2 & 481 & 90 \\
98 & $1,543.6$ & 3.2 & 482 & 92 \\
99 & $1,545.8$ & 3.2 & 483 & 91 \\
100 & $1,547.8$ & 3.3 & 469 & 92 \\
\hline
\end{tabular}

The output spectra of the filter for different values of $R_{\mathrm{r}}$ (radius of structure rods) and $R_{\mathrm{C}}$ (radius of core rods) are shown in Figs. 6 and 7, respectively. It is evident that any increase in the radius of both, leads to red shift of wavelength without any identical change in the amplitude. The detailed specifications of the output wavelengths for
Table 4 Significant parameters of the proposed filter for different values of $R_{\mathrm{r}}$

\begin{tabular}{lllll}
\hline$R$ & $\lambda(\mathrm{nm})$ & $\Delta \lambda(\mathrm{nm})$ & $Q$ & TE $(\%)$ \\
\hline 80 & $1,525.4$ & 2.9 & 525 & 85 \\
85 & $1,533.7$ & 2.8 & 547 & 87 \\
90 & $1,540.4$ & 2.8 & 550 & 88 \\
95 & $1,545.4$ & 2.7 & 572 & 88 \\
100 & $1,550.2$ & 2.6 & 596 & 91 \\
105 & $1,554.7$ & 2.5 & 621 & 91 \\
\hline
\end{tabular}

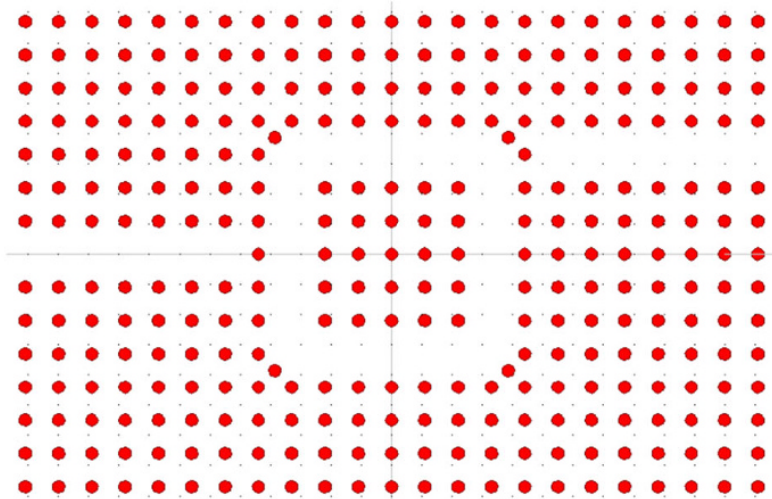

(a)

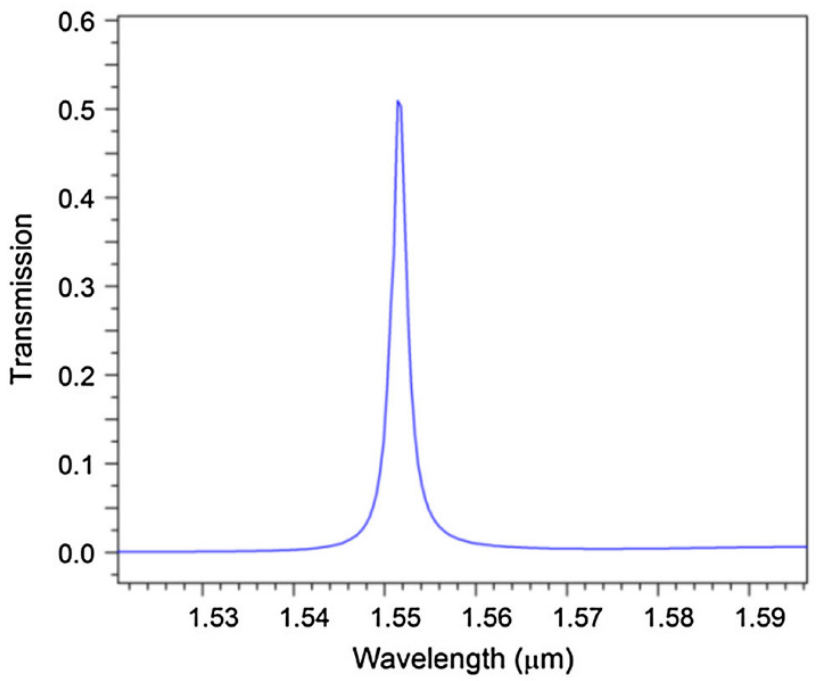

(b)

Fig. 8 a Proposed filter with displaced output waveguide, b output spectra of the filter

different values of $\mathrm{r}$ and $\mathrm{R}$ are listed in Tables 3 and 4, respectively.

In order to study the effect of output waveguide position in the performance of filter, we calculate the transmission spectra for two structures with shifted waveguide and cross waveguide. The results are shown in 


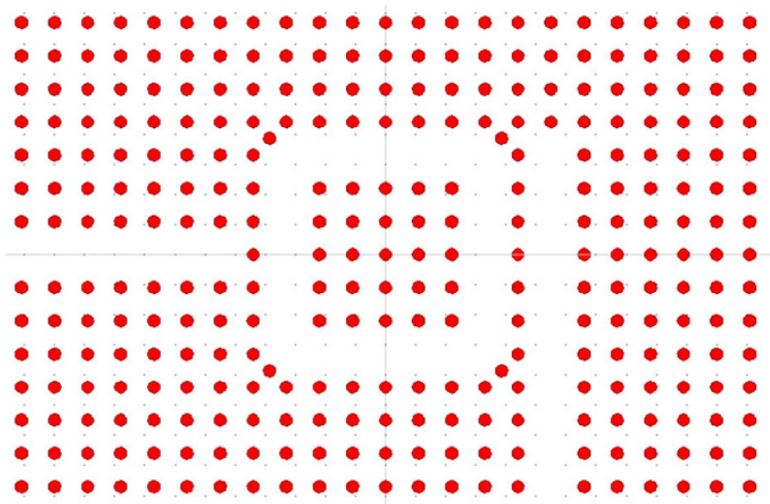

(a)

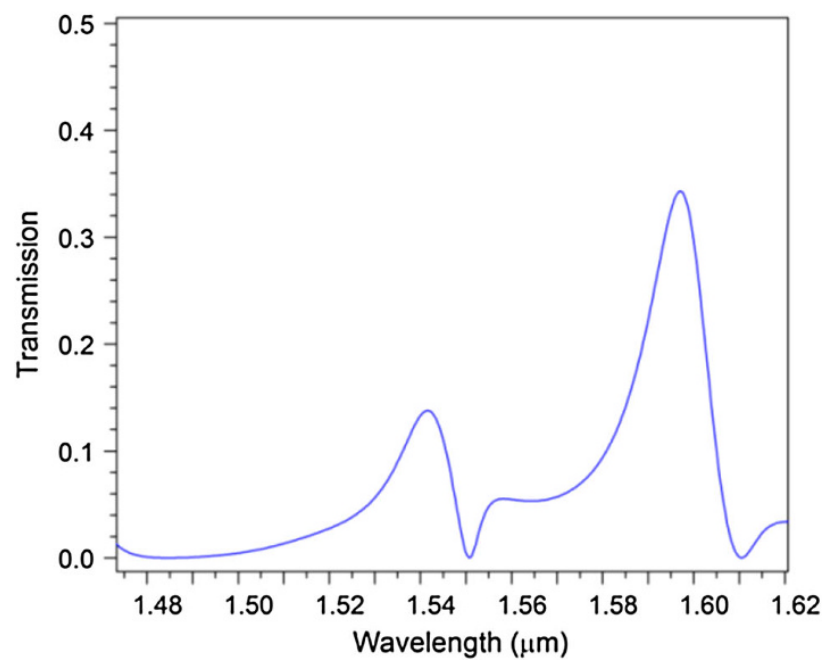

(b)

Fig. 9 a Proposed filter with vertically positioned output waveguide, b output spectra of the filter

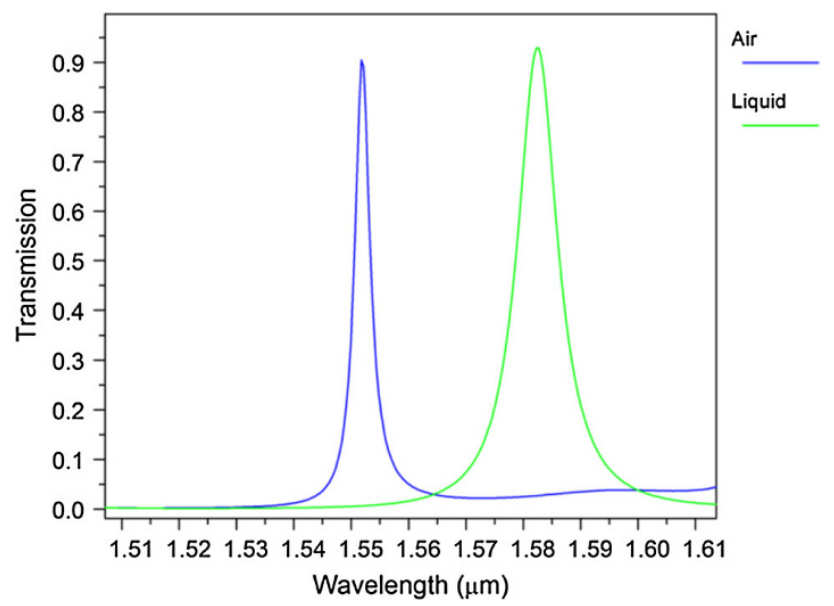

Fig. 10 Output spectra of the filter with liquid crystal as surrounding material of dielectric rods
Figs. 8 and 9. According to the figures, for the case that the output waveguide is shifted up, the transmission coefficient is considerably reduced to about $52 \%$. This can be explained by reflectivity of resonant cavity which acts as a mirror and reflect the beams into the coupling waveguide. While the output waveguide is shifted from the center of resonant cavity, a large part of light within cavity can not be reflected into the waveguide and hence the output intensity falls down. For the case that the output port is vertically positioned, the resonance wavelength is shifted about $60 \mathrm{~nm}$ and its intensity is reduced to about $34 \%$.

We also study the replacement of air as the material surrounding the dielectric rods by liquid crystal. Results are shown in Fig. 10 and it can be seen that using the liquid crystal shifts the peak wavelength to longer values and broadens the spectra. The refractive index of liquid crystal is assumed 1.1.

\section{Conclusion}

In this paper, employing a resonant cavity inside a $2 \mathrm{D}$ photonic crystal we proposed a compact optical filter. The importance of such filter is its very compact design which can be used in integrated optical devices. Using numerical methods such as PWE and FDTD methods we obtained the optical properties of our proposed structure and investigated the effect of different parameters on the output wavelength of the filter. Our results show that by increasing the radius of rods in the structure and also in the core a red shift will occur in the output wavelengths of the filter. Also increasing the refractive index of the dielectric substrate results in a red shift in the output wavelengths of the filter. The total footprint of the filter is less than $76 \mu \mathrm{m}^{2}$, this shows that our filter is compact enough to be used in all optical integrated circuits. Simplicity of design is the other advantage of our proposed structure.

Open Access This article is distributed under the terms of the Creative Commons Attribution License which permits any use, distribution, and reproduction in any medium, provided the original author(s) and the source are credited.

\section{References}

1. John, S.: Phys. Rev. Lett. 58, 2486 (1987)

2. Liu, Z., Zhou, J., Li, B.: Mod. Phys. Lett. B 27, 1350099 (2013)

3. Yablonovitch, E.: Phys. Rev. Lett. 58, 2059 (1987)

4. Joannopoulos, J.D., Johnson, S.G., Winn, J.N., Meade, R.D.: Photonic crystals molding the flow of light citation. Princeton (1995) 
5. Liu, Wen-Long, Yang, Tzong-Jer: Phys. Lett. A 369, 518 (2007)

6. Liu, W.L., Liou, Y.Y., Wei, J.C., Yang, T.J.: Phys. B 404, 4237 (2009)

7. Johnson, S.G., Fan, S., Villeneuve, P.R., Joannopoulos, J.D., Kolodziejski, L.A.: Phys. Rev. B 60, 5751 (1999)

8. Temelkuran, B., Ozbay, E.: Appl. Phys. Lett. 74, 486 (1999)

9. Mehdizadeh, F., Alipour-Banaei, H.: J. Opt. Commun. 34, 61 (2013)

10. Fan, S., Villeneuve, P.R., Joannopoulos, J.D., Haus, H.A.: Phys. Rev. Lett. 80, 960 (1998)

11. Koshiba, M.: J. Lightwave Technol. 19, 1970 (2001)

12. Qiang, Z., Zhou, W., Soref, R.A.: Opt. Express 15, 1823 (2007)

13. Taalbi, A., Bassou, G., Mahmoud, M.Y.: Optik. 124, 824 (2012)
14. Chen, T., Zheng, C., Li, J.: Mod. Phys. Lett. B 26, 1250170 (2012)

15. Alipour-Banaei, H., Mehdizadeh, F., Hassangholizadeh-Kashtiban, M.: Opt. Quantum Electron. 45, 1063 (2013)

16. Kim, S., Nordin, G.P., Cai, J., Jiang, J.: Opt. Lett. 28, 2384 (2003)

17. Alipour-Banaei, H., Mehdizadeh, F.: Optik. 124, 2639 (2012)

18. Mehdizadeh, F., Alipour-Banaei, H., Serajmohammadi, S.: J. Opt. 15, 075401 (2013)

19. Alipour-Banaei, H., Hassangholizadeh-Kashtiban, M., Mehdizadeh, F.: Optik. 24, 4416 (2013)

20. Johnson, S.G., Joannopoulos, J.D.: Opt. Express 8, 173 (2001)

21. Robinson, S., Nakkeeran, R.: Optik. 123, 451 (2012) 